\title{
A methodological approach to developing and validating IFRS 9 - LGD parameters
}

\author{
Luminita-Georgiana ACHIM \\ Bucharest University of Economic Studies, Bucharest, Romania \\ luminita.achim28@gmail.com \\ Elena MITOI \\ Bucharest University of Economic Studies, Bucharest, Romania \\ elena.mitoi@gmail.com \\ Ioan-Codrut TURLEA \\ Bucharest University of Economic Studies, Bucharest, Romania \\ turleacodrut@gmail.com
}

\begin{abstract}
Since the introduction of the advanced internal rating based approach through the Basel framework, financial institutions and regulators have been dealing with the increased complexity of Loss Given Default models. The development and validation of the parameters has become more formalized and standardized as more prescriptive regulations and guidelines have been published by the European Parliament, European Central Bank and European Banking Authority. Furthermore, following the introduction of IFRS 9 in January 2018 even more emphasis is put on the development and validation as the standard poses new challenges to the way models are designed, developed, validated and increased complexity through the introduction of the lifetime and forward-looking adjustments. This paper address the challenges faced by banks and regulators when assessing and validation the IFRS 9 - Loss Given Default parameters and framework. Moreover, it describes a non-exhaustive list of tests that can be performed to establishing the accuracy, discrimination power and stability of the Loss Given Default outputs. It is important that the framework built around the modeling, development and validation process allows models to be easily integrated in the management framework in a flexible manner that can facilitate any changes that must be brought to the models. Hence, this paper outlines a non-exhaustive list of quantitative validation tests considered would satisfy the requirements of the IFRS 9 standard.
\end{abstract}

Keywords: IFRS 9, LGD, challenges, model, validation tests.

\section{Introduction}

The global economic downturn of the last decade highlighted significant limitations of the prevailing IAS 39 incurred loss provisioning framework. In response to the shortcomings of the IAS 39 provisions (the IAS 39 standards were shown to have non-timely recognition of credit loss) the IFRS 9 standard which focuses on developing forward looking expected credit loss estimates was developed. Consequently, regulators and banks have been more and more concerned with the level of the provision coverage and the models used in estimating the expected credit losses, given all layers of the institutions are impacted by the new modeling and validation frameworks developed to accommodate the IFRS 9 requirements.

This paper presents a set of tests that can be performed to validate the IFRS 9 LGD parameter by considering only quantitative methods. The advantage of using statistical methods is that these enable users to easily depict the model's shortcomings. 
Depending on the life cycle phase of the model, the validation activities can be classified as follows:

- Initial validation - performed after the development of the model assesses - if the model is sufficiently robust to pass the approval of the management board and be implemented.

- Periodic validations - are carried after the implementation of the model to assess - if the quality of the model is still adequate or if a refinement or a re-development is needed. This type of validation is usually performed on a yearly basis or when demanded by extraordinary circumstances. Furthermore throughout the life of the model, the monitoring of the model's performance serves as an early warning indicator.

- Monitoring - performed on a more frequent basis (monthly or quarterly) and acts as an early warning system for model deterioration.

The assessment concludes with the drafting of the validation report which provides an overview of the models subject to the validation process, the data used, the tests performed and their outcome. The quantitative elements should a minimum cover accuracy, discrimination, concentration and stability and calibration tests.

When assessing each dimension, thresholds should be assigned to each test in accordance with the regulatory requirements and risk appetite of the institution. The thresholds can be further associated with a visual representation, the most often used, being the traffic lights approach:

- Green - the model is performing as expected,

- Amber - the performance of the model is satisfactory and additional investigations should be performed to understand the limitations presented by the tests and how they can be overcome,

- Red - the performance of the model is unsatisfactory the model is inadequate for its purpose/use or its performance has decreased significantly, hence refinement or replacement is necessary.

The validation report's outcome is the basis for the recommendations and actions to be undertaken to remediate the identified issues, hence it is important to contain a clear distinction between degradation, inadequacy and limitations of the model. The validation considerations should start from the model developing phase, when the data must be split in order to ensure the independence in terms of development and validation.

For IFRS 9 purposes, the validation sample should meet the out of time and out of sample criteria to ensure that the model's performance is representative and appropriate for the entire population.

Best practices suggest that the development and validation samples are split using:

- Random selection without replacement: when the institution has a large sample available, it is usually split into $80 \%$ when the model is developed and the remaining $20 \%$ is used for validation purposes. However, the following conditions must be met: the two samples must be homogenous in terms of underlying risk drivers and characteristics in order to ensure comparability, furthermore initial sample should be large enough to ensure an efficient random selections algorithm.

- Cross-validation (Bootstrapping): the method is recommended in the case of low sample sizes. By applying these techniques multiple validation samples are created by extracting with replacement from the initial validation sample. The number of samples created depends on the data and model characteristics. The tests are performed on each sample and the result is determined by computing the average of the outputs. 
Under both considerations the validation sample should be representative of the current portfolio characteristics furthermore the consistency of the definition of default should be ensured.

\section{Literature review}

The IAS 39 framework was based on the estimation of provisions on an incurred losses basis, hence losses would have been identified based on from past events. Hence, the implications of

PICBE | 685 expected future credit loss events were not considered. Moreover, the approach was criticized due to the heterogeneity of approaches and the lack of visibility over future conditions that would make the decision making process of stakeholders and users of financial statements mode difficult (Magnan and Markarian, 2011).

Azzaz et al. (2015) have raised their concerns with regard to the pro-cyclical nature of the IAS 39 standard which can lead to a snowball effect and impact the real economy in times of stress reflective of a downturn.

IFRS 9 has been introduced by regulators in order to reduce this effect and ensure for a timely identification of losses.

The basis of any IFRS 9 model is the definition of default, however IFRS 9 (B5.5.37 of [IASB, 2014]) does not provide guidance on the events that would be considered default, the standard leaves it up to the entities to consider what would be in their case an appropriate definition of default that is consistent with internal credit risk practices. A model of default that was used is the one depicted by Lando (1998) presenting a reduced-form model of default, which provides a framework for determining a robust proxy for credit risk through the intensity of default. The Lando model is based on incurred or almost sure losses. This mechanism has been criticized by the many which recommended to explore some more forward-looking alternatives.

Hence this led to the need of exploring the use of forward looking information and setting expected credit losses (ECL) in order to overcome the weaknesses identified for the IAS 39 framework. This approach advocates for the recognition of loss allowances.

Moreover the IFRS 9 advocates debate on whether credit ratings could be used as a source of information for the assessment of riskiness of debtors, however if we were to think of external rating agencies their ratings are backward-looking.

Furthermore in addition to the limitations linked to the accuracy of the ratings and their backwards-looking nature, the lack of timeliness is considered the main drawback, (Cheng and Neamtiu, 2009) and (Bolton et al., 2012). Additional concerns are raised also with regard to downgrading process being too slow leading to inefficiency (Morgenson, 2008) not the possibility to predict bankruptcies (Buchanan, 2009).

This together with the IFRS 9 recommendations make the ratings less valuable, as presented earlier, the latter encourages considering changes in market indicator. Therefore, it is important to develop market-based measures of default risk to give a point-in-time indication of creditworthiness of an entity of interest.

One of the main differences compared to the previous standard is that defaulted assets as well as assets that have deteriorated their performance require life time provisions.

One of the main challenges introduced by the IFRS 9 standard requires the incorporation of macroeconomic variables into the computation of the expected credit losses (IFRS 9.5.5.17(c), B5.5.49-54). Hence, from a modeling point of view in addition to the computation of lifetime expected credit losses the other challenge is to incorporate macroeconomic variables such as 
Gross Domestic Product (GDP), inflation into the models to increase the accuracy of forecasts ensuring the products respond to the market conditions.

With respect to the IFRS 9 LGD requirements it is expected that in the case the institution has been using an LGD model for the Advanced Internal Rating Based (A-IRB) approach under the Basel requirements, adjustments are made to the parameters to ensure that the IFRS 9 LGD does not contain indirect costs or a downturn component (IFRS 9.5.5.17-20, B5.5.49-54, BC5.283).

From an integration and interconnection, institutions have to proactively align their regulatory capital, economic capital, stress testing and now expected credit losses frameworks and ensure the development of an integrated approach, furthermore it is recommended that institutions align their stage 2 allocation to the risk appetite in order to prevent high risk portfolio going unnoticed based on a stage 2 allocation deficiency.

Supervisors and audit companies like noticed that due to time pressure of implementing the IFRS 9 models and the fact that the standard does not stipulate the necessity of model validation, many institutions have ignored the validation of models or have only leveraged methods and tests used for other frameworks which are not completely appropriate for the IFRS 9 purposes.

Hence, the Global Public Policy Committee (GPPC) issued in June 2016 a document providing guidance of the impairment requirements presenting examples of non-compliance with IFRS 9 as well additional clarifications on the governance arrangements and the expectations on the validation process.

Further clarifications have been provided by the European Banking authority, the EBA/GL/2017/06 published in 2017 requires in accordance with "Principle 5 - ECL model validation" the validation, monitoring and review of the modeled parameter as well as the identification of the model's limitations also putting emphasis on the independence of the validation process. This principle is in line with the Bank for international settlement's requirements presented in Guidance on credit risk and accounting for expected credit losses published in December 2015.

Due to the elements presented above, the validation of credit risk parameters becomes an essential component of any institution's framework. In order to ensure the quality of the assumptions and methods used in estimating the parameters used in the expected credit loss computation, especially since it directly impacts the financial institution's profit, as such the proper understanding of the model, its accuracy, robustness and limitations will ensure the institution's profitability and competitiveness.

\section{Methodology}

The research methodology is based on a deductive approach which highlights from a theoretical perspective the development of an IFRS 9 compliant LGD parameter as well as the associated validation approaches.

As research method is based on performing an in depth analysis of specialized literature and accounting requirements in order to identify the relevant work on the analyzed subject.

The academic community has mostly focused on the assessment of the LGD from the Basel perspective, hence from the point of view of the estimation of the unexpected losses and the derivation of the risk weighted assets or the assessment of the LGD model from a stress testing perspective. 
Previous analysis such as Weij and Hollender (2009), Yang and Tkachenko (2012) would have focused on presenting Basel compliant models. While in more recent year researches such as Gaurav et all (2016) would have started looking into the point in time and forward looking assessments compliant with stress testing frameworks and IFRS 9 expected credit losses.

Hence previous studies would have only focused on one type of model and assessed its implementation, while the current study presents a comparison between the different methodologies and how they can be tailored on different portfolios depending on data availability and the complexity of model.

Overall the research is addressing the difficulties faced in practice by institutions that in the past would have opted to use the normative, CRR prescribed LGD values as data would have not been available or there would have been significant burden and additional costs incurred by those institutions if they would have developed their own LGD estimates.

However, with the introduction of the IFRS 9 standard financial institution are encouraged to develop their own loss estimates based on information available (inclusive of forward looking information) with undue cost or effort.

The main objective of the paper is to present a conceptual framework presenting modelling techniques and validation tests that financial institutions can use in the development of IFRS 9 models depending on the available data in order to comply with the IFRS 9 requirements.

The authors have presented what in their opinion represent the most appropriate models for developing IFRS 9 compliant LGD models as well as the tests that can be used to validate the accuracy of the estimates.

The paper is based on a qualitative research of the expectations set by international organizations (European Banking Authority (EBA), Basel Committee on Banking Supervision (BCSB)) concerning the IFRS 9 LGD parameters well as other academic papers as previously presented in the literature review section and over this chapter. The following sub-chapters present the authors view on the modelling approaches for LGD parameters and how they can be implemented.

The research was constructed by analysis previous studies and statistic models observing the limitations of the analysis especially around the ways data is used in the development of LGD models as well as the specific IFRS 9 requirements around the LGD parameter.

\section{Results and discussions}

Over this sub-chapter comparison of three models that institutions could use in practice to meet the IFRS 9 requirements. The research starts with the presentation of the least complex model, namely the Market based LGD, while also requires the least amount of data.

The second model is based on vintage analyze, hence more information would be required as well as more complex modeling techniques. The last model is the most complex and would be the development of a score card hence the institution would build a grading structure and calibrate the LGD for each of the individual grades.

The last section of the research presents the main tests used to validate the methodologies.

\section{LGD types under IFRS 9}

The validation framework of the LGD Parameter should ensure recoveries are accurately reflected in the model and the workout process is adequate, this has to be backed by appropriate internal governance arrangement and documentation.

In terms of workout process the LGD can be split in two components: loss given cure 
(LGC) and loss given loss (LGL), however some institutions also include a third component loss given restructuring (LGR). Furthermore, the LGD parameter must take into account cure and liquidation events as well as the integration of forward looking elements. Furthermore, the differentiation between IFRS 9 LGD, IRB (Internal Rating Based) and expected loss best estimates (ELBE) must be made, as IRB and ELBE are usually derived from IFRS 9 LGD, however separate validation exercises must be performed for the ELBE parameters as these must satisfy Capital Requirements Regulation (CRR) requirements that are not applicable to IFRS 9 LGD. ELBE estimates must be a measure of economic loss to satisfy the requirements of CRR art. 5 par. 2, while IFRS 9 LGD estimates must measure accounting loss.

Depending on the way the LGD model was built the validation techniques used can differ significantly, hence the next part of the paper will present four options of building LGD models and the statistical tests used for their LGD validation.

Based on best practices, in terms of the recovery component the following elements are expected to be presented in an LGD model:

- Secured recoveries from collateral;

- Unsecured recoveries from payments;

- Direct costs of the recovery process;

- Months in default, respectively time to sell of collateral of time to full recovery of the outstanding amount;

- Discounting of recoveries.

\section{Market value based LGD}

This LGD model is based on the market value estimation and is used for portfolios secured by real estate with limited data history or poor data quality. The model is based on the adjusted value of collaterals the market value of the collateral is adjusted using a benchmark or proxy haircut and time to sell depending on the collateral type. Given the method is not based on historical recoveries it cannot be classified as a statistical method.

A limitation of this method is that unsecured recoveries are not integrated hence there is no clear distinction between the secured and unsecured collaterals leading to potentially overestimated LGD.

The formula for the market value LGD is as follows:

$L G D=\operatorname{MAX}\left[L G D_{\text {Min }} \frac{E A D-\left(\frac{M V(1-H C)-D R C}{(1+E I R)^{T T S}}+\frac{U R}{(1+E I R)^{T T R}}\right)}{E A D}\right]$

where:

$L G D_{\text {Min }}$ - Minimum regulatory imposed LGD;

EAD - exposure at default;

$\mathrm{MV}$ - market value of the collateral;

$\mathrm{HC}$ - haircut value applied to the market value;

DRC - direct recovery cost;

EIR - effective interest rate;

UR - unsecured recoveries;

TTS - time to sell;

TTR - time to recovery. 
Based on best practices the HC, TTS, TTR, UR parameters are computed using simple or EAD weighted averages segmented according to the client, product and collateral type.

It is recommended that outliers are clustered into grades $5 \%$ and $95 \%$, quantile representing the most common outliers selected to be grouped in different grades $s$ to avoid $t$ biases in the rest of the estimation. There are certain types of collaterals which due to their nature or improbability of recoveries are subject to a qualitative assessment performed by an expert. When using this approach, special attention must be given to the frequency collaterals re-evaluation and the estimation of the applied haircuts and from a validation perspective back-testing of the accuracy of the expert's assessments against observed outcomes.

If statistical models based on pools or grades are used for the estimation of HR, UR, TTS, TTR, than the discrimination, stability and concentrations test can be performed.

\section{Vintage models}

Another method for the computing the LGD is by using vintage models based on two dimensions: the vintage the default date of the pool of accounts analyzed and the number of months in default limited to the workout period length (2, 3 years depending on the institution's practices). It must be ensured that there are no vintages with 0 defaults and each vintage is representative for the estimation, hence no outliers are identified in the vintage groupings. In practice, institutions try to capture all recoveries and identify the moment when the recoveries become immaterial to prevent having unlimited months in default.

Another issue is the estimation of the incomplete recoveries institutions are most of the time unable to compute the recovery values for more recent periods. In such cases, the most commonly used method is extrapolation. Institutions should not exclude most recent vintages hence incomplete outcomes, as this would lead to not observing the most recent recovery behavior.

\section{Non-parametrical LGD models}

Another method to estimate the LGD is by using a non-parametrical classification method, such as decision trees. In practice, the most commonly used model is Chi-square automatic interaction detection (CHAID) developed by Kass in 1980 based on the following steps must be undertaken:

- Computation of ex-post facility/client level LGD based on recoveries, costs and exposure at default;

- Identification of a set of explanatory variables that can discriminate the ex-post results such as: collateral type, revaluation date, industry type, granted limits, exposure at default, months on book, months to maturity. The variables are ranked based on their clustering power using a mix of qualitative methods, like applying expert judgment, and quantitative analysis (ANOVA);

- Construction of the decision tree using the selected list of explanatory variables in order to ensure a high discrimination between the different leafs of the decision tree.

\section{Score card}

The forth method is to develop a scorecard using a logistic regression, linear regression or neutral networks. The following issues can be encountered when developing a LGD scorecard:

- The LGD is a continuous variable, as such a conversion to a binary variable must be performed and, furthermore, the scorecards can generate higher estimation errors than simple averaging methods The Market Value LGD. 
- The length of the outcome period is directly dependent on the length of the workout period.

\section{Statistical tests for LGD Validation}

The following part of the paper will describe the most commonly used test for the validation of LGD.

If the development sample isn't large enough the t test can be performed. The t-test is based on three assumptions: independence between observations, normal distribution of the observed LGD (a sample is expected to be normal distributed if the sample is above 30 observation) and equal variance of the observed and predicted LGD. The third assumption cannot be validated since the variance of the predicted LGD is unknown.

A one sample t-test compares the sample mean with a known mean. In this case we compare the observed LGD (sample mean) with the predicted LGD. The absolute observed mean of LGD is computed in the following way:

$$
L G D_{\text {observed }}=\frac{\sum_{i=1}^{n}\left(L G D_{\text {observed }}(\%)_{i} * E A D_{i}\right)}{n}
$$

Likewise, absolute predicted mean of LGD is given by the following formula:

$$
L G D_{\text {Predicted }}=\frac{\sum_{i=1}^{n}\left(L G D_{\text {Predicted }}(\%)_{i} * E A D_{i}\right)}{n}
$$

where:

$L G D_{\text {observed }}(\%)_{i}$ - LGD observed for client/facility "i";

$L G D_{\text {Observed }}(\%)_{i}$ - LGD predicted assigned to client/facility "i";

$E A D_{i}$ - Exposure at default for client/facility "i".

$\mathrm{n}$ - Sample size;

The hypotheses of the t-test are:

$H_{0}-L G D_{\text {Predicted }}=L G D_{\text {observed }}$;

$H_{1}-L G D_{\text {Predicted }}<>L G D_{\text {Observed }}$.

The one-sample T-Test value is calculated as follows:

$t=\frac{L G D_{\text {Observed }}-L G D_{\text {Predicted }}}{\frac{\sigma}{\sqrt{n}}}$

where:

$\sigma$ - The standard deviation of $L G D_{\text {Observed }}$;

$\mathrm{n}$ - Sample size.

In order to accept or reject the null hypothesis, the value of the t-test is compared with the critical value of the t-test distribution table based on a confidence internal and degrees of freedom (usually a $\alpha=0.05$ and $n-1$ degrees of freedom). In case of a one-tailed t-test the LGD's underestimation is tested, while is a two tailed t-test is performed the LGD is tested for both under and over-estimation. The test has to be performed at a segment level. Furthermore, the result of the t-test performed on the development sample is compared to the result of the validation sample to identify any decrease of the model's power.

Regardless of the size of the sample, the Mean Absolute Error (MAE) can be computed to assess the calibration of the LGD model. MAE represents the absolute difference between the realized and estimated LGD. The test should be performed both at a portfolio and segment level: 


$$
M A E=\sum_{i=1}^{n} \frac{\left|L G D_{i}^{R}-L G D_{i}^{E}\right|}{n}
$$

where:

$L G D_{i}^{R}$ - realised LGD calculated for facility $\mathrm{i}$;

$L G D_{i}^{E}$ - estimated LGD for facility i;

PICBE |

$n$ - total number of facilities.

Another test that can be performed to assess the LGD calibration is the Loss Shortfall (LS) ration representing the total difference between the observed and the estimated LGDs to the total observed LGDs. The test can be performed both at portfolio and at segment level:

$L S=1-\frac{\sum_{i=1}^{n} L G D_{i}^{E}}{\sum_{i=1}^{n} L G D_{i}^{R}}$

where:

$L G D_{i}^{R}$ - realised LGD calculated for facility I;

$L G D_{i}^{E}$ - estimated LGD for facility $\mathrm{i}$;

$n-$ total number of facilities.

A test used to assess the discrimination power of the model is Welch's test the unequal variance t-test which assesses if two populations have equal means. One of the main advantages over the t-test is that it as can be used on samples that have unequal variances and sample sizes. Hence, the t-test is often used for calibration, while Welch's test is used for assessing the discriminatory power of the LGD model in the live environment, hence the model's performance on the development sample and the validation sample must be compared. The following steps can be performed:

- Comparison of the absolute predicted mean of LGD between pools:

$$
t=\frac{\text { LGD }_{\text {Predicted }_{i}}-\text { LGD }_{\text {Predicted }_{j}}}{\sqrt{\frac{\sigma_{i}^{2}}{N_{i}}+\frac{\sigma_{j}^{2}}{N_{j}}}}
$$

- Comparison of the absolute observed mean of LGD between pools:

$$
t=\frac{\text { LGD }_{\text {Observed }_{i}}-L G D_{\text {Observed }_{j}}}{\sqrt{\frac{\sigma_{i}^{2}}{N_{i}}+\frac{\sigma_{j}^{2}}{N_{j}}}}
$$

where:

$\mathrm{I}, \mathrm{j}$ - the two pools of the segmentation;

$\sigma-$ Standard deviation;

$\mathrm{N}$ - Sample size.

The degrees of freedom (DF) used to obtain the $t$ critical value is computed as follows:

$$
D F \approx \frac{\left(\frac{\sigma_{i}^{2}}{N_{i}}+\frac{\sigma_{j}^{2}}{N_{j}}\right)^{2}}{\frac{\sigma_{i}^{4}}{N_{i}^{2} D F_{i}}+\frac{\sigma_{j}^{4}}{N_{j}^{2} D F_{j}}}
$$


where:

$D F_{i}=N_{i}-1$ and $D F_{j}=N_{j}-1$.

Another method for assessing the discrimination of an LGD model is the ROC curve. However, as the LGD is not bound to a $[0,1]$ interval as in the case of PD, the following considerations have to be taken into account when computing the cumulative distribution functions (CDFs):

- In practice, institutions are more concerned of underestimations, as over estimation might be accepted if argued that the model is more conservative and the overestimation covers for potential data quality issues or modeling limitations.

- The institution has to define the good and bad events and to compute a binary variable based on the comparison of observed and estimated losses.

- The data is sorted ascending based on the estimated LGD values.

- The CDFs for the bad and good are computed based on the response variables.

- The AUROC is computed and ROC curve is plated on a graph.

In practice it is more difficult to present an aggregated distribution for LGD estimates with regard to bad definition as such it is better to compute the ROC curve on the whole sample. The AUROC must be computed for both the development and validation samples. Compared to PDs LGDs have a lower discrimination power, as such a AUROC of 0.6 is unacceptable for PDs while for LGD it considered acceptable.

\section{Conclusion}

The paper focused on presenting the main IFRS 9 validation expectations as well as challenges faced by institutions with the main at presenting the main LGD modeling processes based on data availability and the institution's modeling capabilities intensity.

In the future, additional considerations should be given to the use of management judgment and overlays, as the IFRS 9 regulatory regime increases accounting judgments since it incorporates forward looking information in the estimation of credit losses which, theoretically, should ensure a timely recognition of provisions. However, as observed since its implementation in 2018, the standard is still at an early stage and more time has to pass for institutions to be able to create robust validation frameworks around the ECL estimates.

Furthermore, the stochastic nature of the new accounting standard, regarding the estimations on expected credit losses (ECLs), the probability of default (PD) and the loss given default (LGD), raises concerns about potential managerial discretion and flexibility of the banks' management teams' effect on banks' financial statements, as well as the moral hazard implied. Hence a robust validation and monitoring framework is required.

The new accounting standard creates many areas of readjustments regarding banks' procedures, processes, evaluation models, benchmark estimators, monitoring and auditing. In order to reassure the successful and harmonized implementation of the new regime, a constant and highly demanding mechanism of governance and controls is required including a robust validation process.

While the paper presents the methodological approaches for addressing data limitations when developing and validating IFRS 9 models as well as the way those models can be validated. Overall, the main limitation of the research is the limited information around the use of management overlays and overrides used in capturing the impact of random events that could impact the macroeconomics, but which cannot be captured using the available models. This 
limitation is driven by the fact that the IFRS 9 standard only came into force in 2018 and previous frameworks from Basel normative capital approaches to economic capital models and stress testing only focused on the estimation of short to medium term forecast, while IFRS 9 aims at estimation the losses over the lifetime of the instruments, hence the difficulty to include and to estimate the impact of the random events. Even though this could be partially overcome by using the Poisson distribution, additional research is required to assess their impact and how they can be captured through management overlays.

Another element that will require further in dept research is the treatment of incompletes, namely those open cases for which the outcome/resolution is yet unknown. Namely those obligors/exposures are classified as stage 3 in accordance with IFRS 9 however there is no information available on the potential loss or no loss that could be associated with the closing of the default. In practice, there are cases where such observations would cure with no loss, hence the increase in cure rate would have an inverse impact on the LGD reduce the LGD estimates, however there would be cases where even though the exposure would cure additional losses would be incurred cure by applying forbearance measures. The institution as well as academia should focus on the assessment of the cure with loss or the estimation of cases that close with a loss.

\section{References}

Azzaz, J., Loisel, S., \& Therond, P. E. (2015). Some characteristics of an equity security nextyear impairment, Review of Quantitative Finance and Accounting, 45, 111-135.

Barth, M. E., \& Landsman, W. R. (2010). How did financial reporting contribute to the financial crisis?, European Accounting Review, 19(3), 399-423.

Basel Committee on Banking Supervision (1988). International Convergence of Capital Measurement and Capital Standards, https://www.bis.org/publ/bcbs04a.htm.

Basel Committee on Banking Supervision (2005). International Convergence of Capital Measurement and Capital Standards: A Revised Framework, BIS, Updated November 2005, https://www.bis.org/publ/bcbs107.htm.

Basel Committee on Banking Supervision (2005a). Validation, Newsletter No. 4., https://www. bis.org/publ/bcbs_nl4.htm.

Basel committee on banking supervision (2015). Guidance on credit risk and accounting for expected credit losses, https://www.bis.org/bcbs/publ/d350.pdf.

Bischof, J., Daske, H. (2016). Interpreting the European Union's IFRS endorsement criteria: the case of IFRS 9, Accounting in Europe, 13(2), 129-168.

Bolton, P., Freixas, X., \& Shapiro, J. (2012). The credit ratings game, The Journal of Finance, 67, 85-111.

Buchanan, M. (2009). Why money messes with your mind, New Scientist.

Carlehed, M., \& Petrov, A. (2012). A methodology for point-in-time-through-the-cycle probability of default decomposition in risk classification systems, Journal of Risk Model Validation, 6(3), 3-25.

Cheng, M., \& Neamtiu, M. (2009). An empirical analysis of changes in credit rating properties: Timeliness, accuracy and volatility, Journal of Accounting and Economics, 47, 108-130.

Edwards, G.A. (2016). Supervisors' key roles as banks implement expected credit loss provisioning SEACEN, Financial Stability Journal, 7(1), 1-25. 
European Banking Authority (2017). Guidelines on credit institutions' credit risk management practices and accounting for expected credit losses.

European Banking Authority (2017). Guidelines on PD estimation, LGD estimation and the treatment of defaulted exposures.

European Banking Authority (2016). Guidelines on the application of the definition of default under Article 178 of Regulation (EU) No 575/2013.

Gaurav, C., Forest, Jr. L. R., \& Aguais, S.D. (2016). Point-in-time loss-given default rates and exposures at default models for IFRS 9/CECL and stress testing, Journal of Risk Management in Financial Institutions, 9(3), 249-263.

Global Public Policy Committee (2016). The implementation of IFRS 9 impairment requirements by banks.

International accounting standards Board (2014). International Financial reporting standard 9 Financial instruments.

Lando, D. (1998). On cox processes and credit risky securities, Review of Derivatives research, 2 , 99-120.

Magnan, M., \& Markarian, G. (2011). Accounting, governance and the crisis: is risk the missing link?, European Accounting Review, 20, 215-231.

Morgenson, G. (2008). Debt watchdogs: Tamed or caught napping?. New York Times.

Pool, S., De Haan, L., Jacobs, J.P. (2015). Loan loss provisioning, bank credit and the real economy, Journal of Macroeconomics, 45(1), 124-136.

Reitgruber, W. (2015). Methodological thoughts on expected loss estimation for IFRS 9 impairment: hidden reserves, cyclical loss predictions and LGD back testing, Credit Technology by Serasa Experian, 92, 7-29.

Temim, J. (2016). The IFRS 9 impairment model and its interaction with the Basel framework, Moody's Analytics Risk Perspectives, 8(1), 13-33.

Weij, W., \& Hollander, M. (2009). Improving PD and LGD models: following the changes in the market, SNS Real, Utrecht.

Yang, B.H. (2017). Point-in-time PD term structure models for multi-period scenario loss projection: methodologies and implementations for IFRS 9 ECL and CCAR stress testing, Journal of Risk Model Validation, 11(3), 1-17.

Yang, B. H. \& Tkachenko, M. (2012). Modeling exposure at default and loss given default: empirical approaches and technical implementation, The Journal of Credit Risk, 8(2), 81-102. 